\title{
Improvement of the adhesion strength between copper plated layer and resin substrate using a chemically adsorbed monolayer
}

\author{
K. Tsuchiya*, T. Ohtake, K. Ogawa \\ Department of Advanced Materials Science, Graduate School of Engineering, Kagawa University, \\ 2217-20, Hayashi-Cho, Takamatsu, Kagawa, 761-0396, Japan \\ E-mail:s12g566@stmail.eng.kagawa-u.ac.jp
}

\begin{abstract}
With reducing the size and weight of electric devices, high-tensile, light and fine copper wire is demanded. So the production technique of a copper wire plated on a super fiber resin (Vectran film) was researched for improving the adhesion strength between the copper and the resin. In this study, we used the $\mathrm{Cu}^{2+}$ or $\mathrm{Pd}^{2+}$ complex prepared with a chemically adsorbed monolayer (CAM) to improve the adhesion strength between the copper plated layer and the Vectran film. As the result of scotch tape test, it was observed that the adhesion strength between the copper plated layer and Vectran film was improved by the $\mathrm{Cu}^{2+}$ or $\mathrm{Pd}^{2+}$ complex CAM.
\end{abstract}

\section{Introduction}

Currently copper wires are used in wide field, such as electric devices, electric components, wire harness for electric appliances and automobiles, and electric power cable. The down-sizing of the electric devices are recently remarkable. So, high-tensile, light and fine copper wires are demanded. Nevertheless, the current copper wires become finer, the tensile-strength become weaker. To solve this problem, the production technique of copper wire, which is formed with copper plated on the surface of a super fiber, is researched [1]. Although the super fiber is a high strength and high modulus polymer resin, adhesion strength between the metal and the resin is weak due to low surface energy of the resin, and the copper plated layer on the resin is easily peeled off [2]. Previously, anchor effect was used to improve the adhesion strength. But that technique needed injurious agent, such as hexavalent chromium, to generate irregularities on the resin surface [3].

In this study, we used a chemically adsorbed monolayer (CAM) containing imidazole groups as an interlayer between the copper plated layer and the resin surface to improve the adhesion strength. CAMs were formed with covalent bonds binding with the resin surface having hydrophilic functional groups. In addition, the imidazole group is known to form complex bonds with copper. Thus the copper plated layer and the resin are chemically bonded through the complex monolayer.

\section{Materials and Methods}

In this study, we employed the chemical adsorption (CA) technique in order to prepare CAM terminated with imidazole group. Figure 1 shows molecular structure of Imidazole Silane (IM-1000, JX Nippon Mining \& Metal Inc.) which was used to prepare CAM as a chemical adsorbent. It is a silane coupling agent which has an imidazole group and some alkoxyl groups.

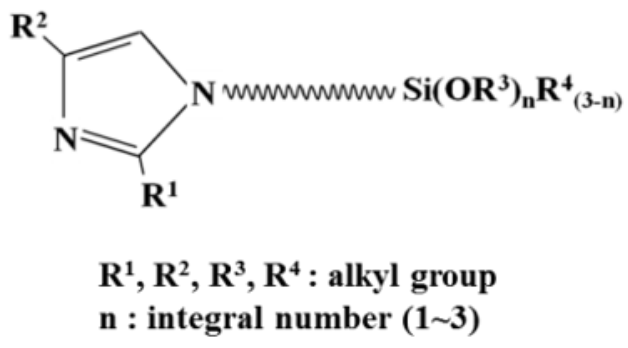

Fig. 1. Molecular structure of Imidazole Silane

In this study, we used Vectran (VECSTAR, KURARAY CO., LTD.) as a resin substrate. Vectran is a polyarylate super fiber. Figure 2 shows the molecular structure of Vectran. And we especially used the Vectran film rather than the Vectran fiber, because the film is useful for easily analysing the adhesion strength between the copper plated layer and the Vectran film.

Vectran films were washed with acetone (Wako Pure Chemical Industries, Ltd.) and $95 \%$ ethanol (SOLMIX, Japan Alcohol Corporation) with ultrasonic bath. The 
experimental process and all the sample conditions prepared in this study are shown in Fig. 4.

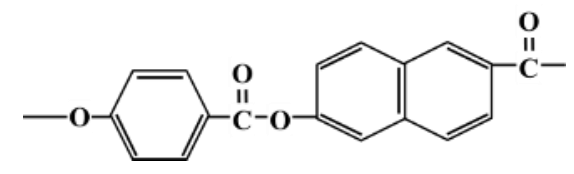

Fig. 2. Molecular structure of Vectran

\subsection{Oxygen plasma treatment}

Hydrophilic functional groups on substrate surface are necessary to prepare CAM. So we treated the Vectran film with oxygen plasma (Plasma Cleaner SAMCO PC$300 \mathrm{~K}$, SAMCO INC.) to introduce the hydrophilic functional groups at the surface of the Vectran film. After evacuation below $5.0 \times 10^{-5} \mathrm{~Pa}$, oxygen gas was introduced into the plasma system. The pressure and the flow rate of the oxygen gas were regulated to be $5 \mathrm{~Pa}$ and $12 \mathrm{sccm}$, respectively. RF power of $250 \mathrm{~W}$ was applied to generate oxygen plasma for $1 \mathrm{~min}$. In Fig. 4, sample A indicates the Vectran film after the treatment with the oxygen plasma.

The changes of the Vectran film surface by the oxygen plasma treatment were evaluated by atomic force microscope (AFM; scanning probe microscope JSPM420, JEOL Ltd.)

\subsection{Preparation of Imidazole Silane CAM}

Firstly, Imidazole Silane was diluted at 3 wt $\%$ with propylene glycol. Moreover, this diluted solution was diluted at 1: 20 with $99.5 \%$ ethanol (Wako Pure Chemical Industries, Ltd.) for preparing the chemical adsorption solution. Next, the Vectran film treated with the oxygen plasma (sample A) was dipped in the chemical adsorption solution for $2 \mathrm{~h}$. Then alkoxyl group in the Imidazole Silane changed to silanol group with water, and the silanol group act to form covalent bond with the hydrophilic functional group on Vectran film by dehydration condensation. Fig. 3 indicates the scheme of the chemical adsorption of the Imidazole Silane. After that, the Vectran film covered with ISCAM was washed with $95 \%$ ethanol and kept in the air atmosphere for $24 \mathrm{~h}$. After $24 \mathrm{~h}$, this Vectran film was washed with acetone and $95 \%$ ethanol in the ultrasonic bath. Sample B in Fig. 4 indicates that the Vectran film covered with IS-CAM.

Adsorption of Imidazole Silane on the Vectran film was confirmed by X-ray photoelectron spectroscopy (XPS; PHI 5000 VersaProbe TM, ULVAC-PHI, Inc).

\subsection{Preparation of $\mathrm{Cu}^{2+}$ or $\mathrm{Pd}^{2+}$ complex with imidazole groups}

In this study, $\mathrm{Cu}^{2+}$ or $\mathrm{Pd}^{2+}$ complex were prepared with the imidazole groups of IS-CAM prepared on the surface of the Vectran film to improve the adhesion strength between the copper atoms of the copper plated layer and the imidazole groups of IS-CAM.

The Vectran film covered with IS-CAM (sample B) was dipped in $0.01 \mathrm{M} \mathrm{CuCl}_{2}$ aqueous solution for $3 \mathrm{~h}$ to form $\mathrm{Cu}^{2+}$ complex with the imidazole groups. After that, this Vectran film was washed with pure water with ultrasonic bath. This Vectran film is shown as sample C in fig. 4.

On the other hand, the Vectran film covered with ISCAM (sample B) was dipped in $0.01 \mathrm{M} \mathrm{PdCl}_{2}$ aqueous solution for $3 \mathrm{~h}$ to form $\mathrm{Pd}^{2+}$ complex with IS-CAM. After that, the Vectran film was washed with $1.0 \times 10^{-3} \mathrm{M}$ $\mathrm{HCl}$ with ultrasonic bath. This Vectran film is shown as sample D in fig. 4.

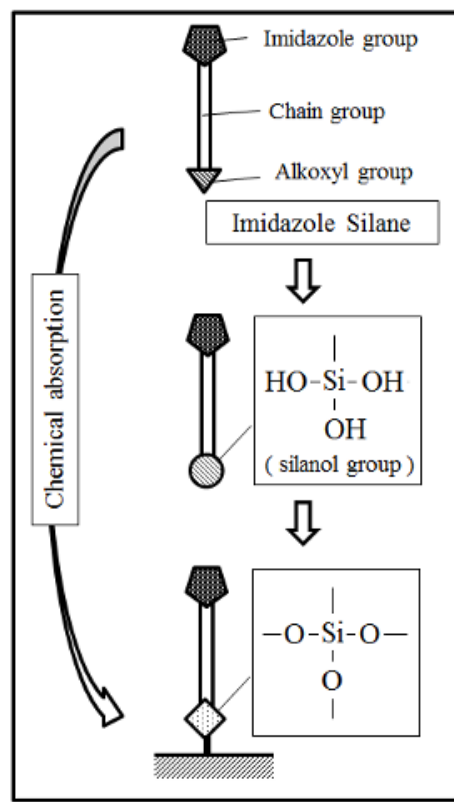

Fig. 3. Chemical absorption scheme of Imidazole Silane molecule
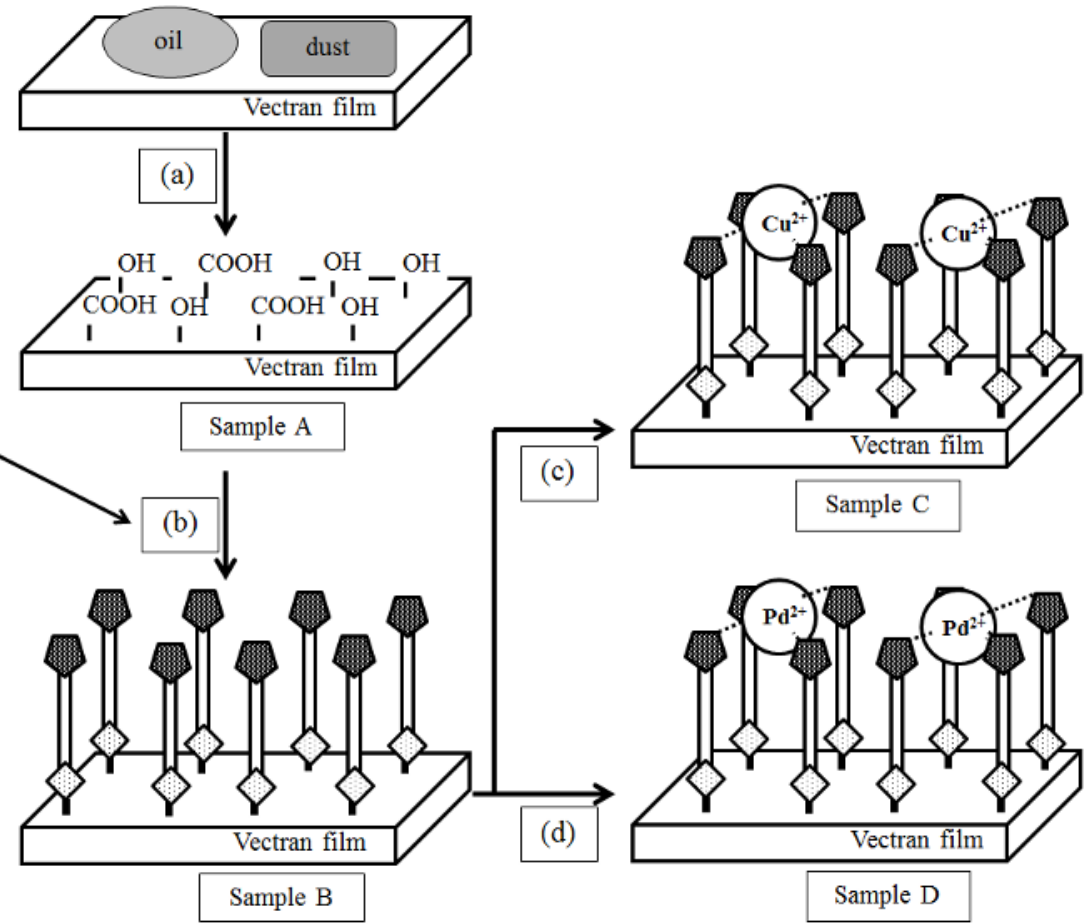

Fig. 4. Sample preparation; (a) ultrasonic cleaning and oxygen plasma treatment, (b) formation of IS-CAM by CA technique, (c) formation of $\mathrm{Cu}^{2+}$ complex with IS-CAM, (d) formation of $\mathrm{Pd}^{2+}$ complex with IS-CAM 
The formation of $\mathrm{Cu}^{2+}$ or $\mathrm{Pd}^{2+}$ complex with imidazole groups was evaluated by XPS.

\subsection{Electroless copper plating and Scotch tape test}

In this study, sample A, B, C, D and raw Vectran film were electroless copper-plated. Firstly, all samples were alternately dipped twice in $\mathrm{SnCl}_{2}$ aqueous solution (100mL/L Sensitizer S-10X, C.Uyemura \& Co.,Ltd.) and $\mathrm{PdCl}_{2}$ aqueous solution $(100 \mathrm{~mL} / \mathrm{L}$ Activator A-10X, C.Uyemura \& Co.,Ltd.). The temperature of these aqueous solutions and the dipping time were $25{ }^{\circ} \mathrm{C}$ and 1 min, respectively. Secondly, these samples were dipped in the electroless copper plating solution (THRU-CUP

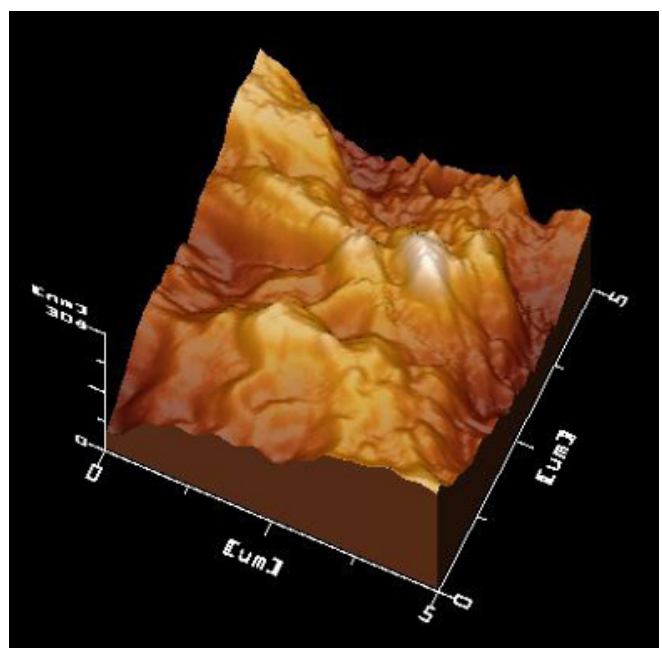

before the oxygen plasma treatment
PEA, C.Uyemura \& Co.,Ltd.) for $3 \mathrm{~min}$ at $30^{\circ} \mathrm{C}$.

Finally, the adhesion strength between the copper plated layer and the Vectran film was evaluated by scotch tape test. Scotch tape (Scotch ${ }^{\circledR}$ BK-12, 3M Company) adhered to the copper plated layer was peeled off. After that, we observed whether the copper plated layer was peeled off or not by using the optical microscope (MX-50, Olympus corporation).

\section{Result and discussion}

\subsection{AFM}

Figure 5 shows AFM 3D images and sectional views of

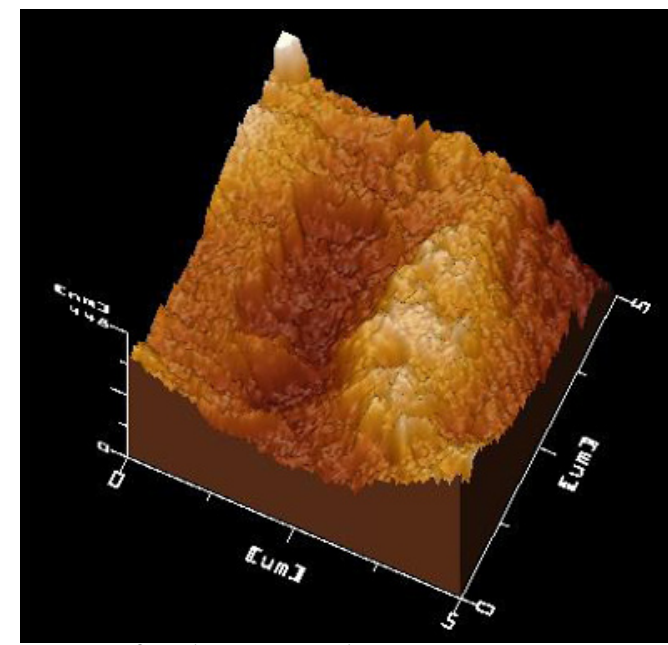

after the oxygen plasma treatment

(a) 3D images of the Vectran film surface

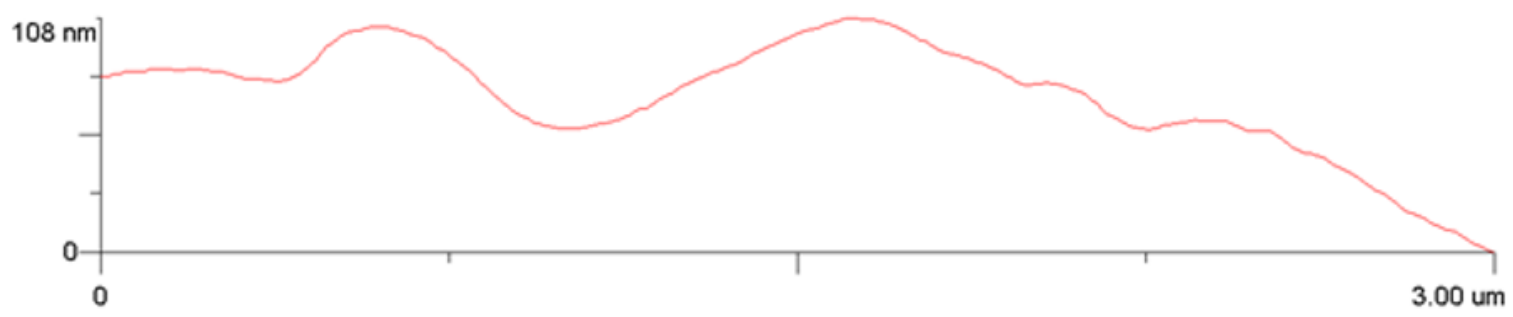

before the oxygen plasma treatment

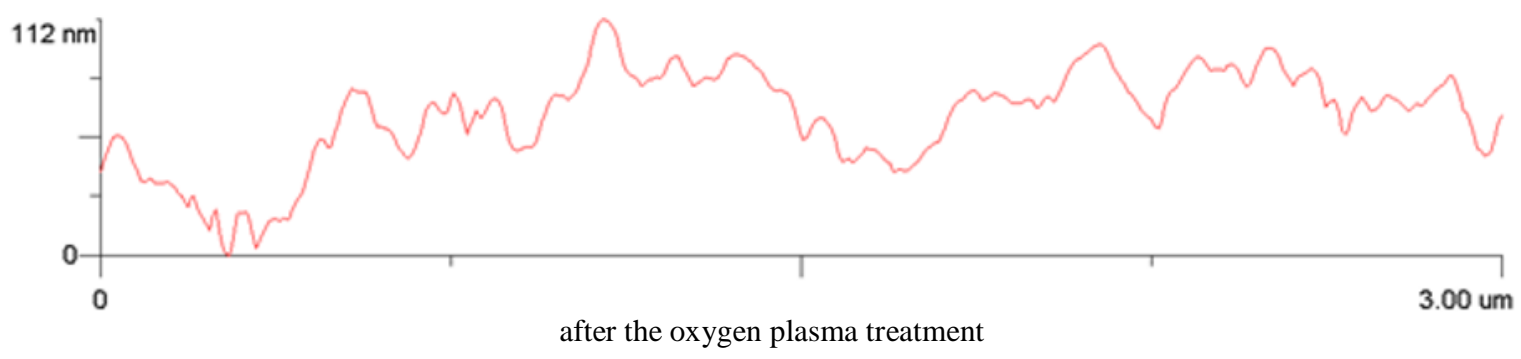

(b) section views of the Vectran film surface

Fig. 5. The AFM images of the Vectran film; (a) 3D images and (b) sectional views before and after the oxygen plasma treatment 
the Vectran film surface. Before the oxygen treatment, the Vectran film surface has a little irregularities. In contrast, many new smaller irregularities were appeared at the surface of the irregularities of the raw film after the oxygen plasma treatment.

\subsection{XPS}

Figure 6 shows the XPS spectra of sample A, B, C, and D. The peaks around $400 \mathrm{eV}$ on the spectra of sample A, B and $C$ represent $N(1 s)$ [4]. But that $N(1 s)$ peak didn't appear on the spectra of sample A, indicating that Imidazole Silane was adsorbed to the Vectran film surface. And the peaks due to $\mathrm{Cu}(2 \mathrm{p} \mathrm{1/2)}$ and $\mathrm{Cu}(2 \mathrm{p}$ $3 / 2$ ) were appered around $930 \mathrm{eV}$ and $950 \mathrm{eV}$ on the spectra of sample C [5], respectively. These indicate that $\mathrm{Cu}^{2+}$ complex was formed with IS-CAM. Moreover, on the spectra of sample D, the peak due to $\mathrm{Pd}(3 \mathrm{~d} 5 / 2)$ was appered around $335 \mathrm{eV}$ [6]. This indicates that $\mathrm{Pd}^{2+}$ complex was formed with IS-CAM.

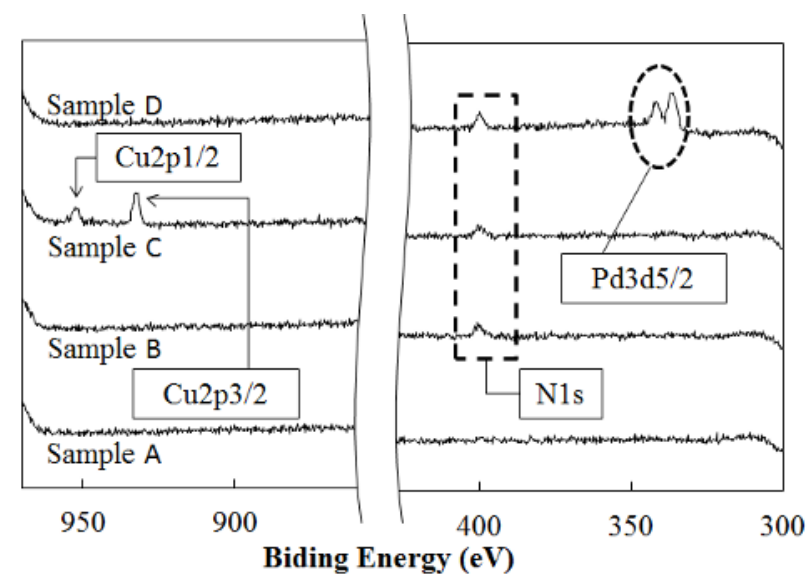

Fig. 6. XPS spectra of sample A, B, C and D

\subsection{Scotch tape test}

Figure 7 shows the optical microscope photographs after the scotch tape tests. (a) is the photograph of the raw Vectran film surface before the scotch tape test. (b) is the photograph of the surface after the scotch tape test of the copper plated layer without the oxygen plasma treatment. (c), (d), (e) and (f) are the photographs of the surface after the scotch tape tests of copper plated layer on sample A, sample B, sample C and sample D shown in Fig. 4, respectively. Most area of the copper plating layer on the raw Vectran film (b) was easily peeled off. While, the peeled off area of the copper plated layer (c) on sample A (only oxygen plasma treatment) was $20 \%$. This improvement may be due to the small surface irregularities and hydrophilicity of the Vectran film surface caused by the oxygen plasma treatment. Moreover, most area of the copper plating layer on sample B (d) wasn't almost peeled off. This may be due to the complex formation between IS-CAM and the copper atoms of the copper plated layer. On sample C and $D(e \& f)$, the copper plated layers were not peeled off at all. These indicate that $\mathrm{Cu}^{2+}$ or $\mathrm{Pd}^{2+}$ complex with
IS-CAM improve the adhesion strength between the copper plated layer and IS-CAM on the Vectran film.
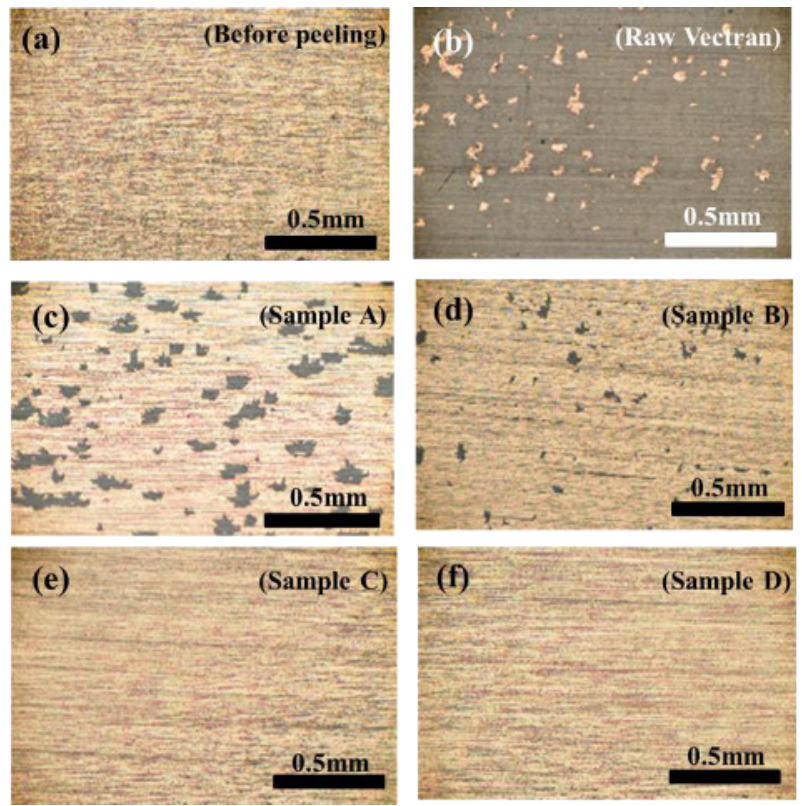

Fig. 7. The optical microscope photographs before and after the scotch tape tests

\section{Conclusion}

In this study, the adhesion strength between the copper plated layer and the Vectran film was improved by using the oxygen plasma treatment, IS-CAM and $\mathrm{Cu}^{2+}$ or $\mathrm{Pd}^{2+}$ complex with IS-CAM. Although the scotch tape test is too simplified method to evaluate the difference of the adhesion, it is clear that formation of the $\mathrm{Cu}^{2+}$ or $\mathrm{Pd}^{2+}$ complex on IS-CAM is effective to improve the adhesion strength between the copper plated layer and the Vectran film. In order to discuss the detail defference between the $\mathrm{Cu}^{2+}$ and $\mathrm{Pd}^{2+}$ complexes, the measurement of peel strength will be needed.

\section{Acknowledgement}

We thank Prof. Naoshi Takahashi of Kagawa University, Takamatsu, Japan for the great support of XPS measurement.

\section{Reference}

1. M. Matsuo, H. Ishikawa, Y. Xi, Y. Bin, Polymer Journal 39, 4 (2007)

2. H. Ishihara, M. Shibaya, Journal of the Textile Machinery Society of Japan 58, 1 (2005)

3. K. Mori, A. Shiro, The Journal of the Surface Finishing Society of Japan 59, 5 (2008)

4. K. Hiasa, Y. Abe, Y. Yoshida, T. Taji, Dental Materials Journal 26, 2 (2007)

5. S. Saito, Y. Araki, M. Taira, Dental Materials Journal 25, 2 (2006)

6. X. Zhao, G. Zheng, I. Tabata, K. Hisada, S. Okubayashi, T. Hori, Sen'i Gakkaishi 62, 3 (2006) 